\title{
On the convergence of the method of successive averages for calculating equilibrium in traffic networks
}

\author{
Richard Mounce \\ Centre for Transport Research, University of Aberdeen, \\ MacRobert Building, King's College, Aberdeen, AB24 \\ 5UA, UK, r.mounce@abdn.ac.uk \\ Malachy Carey \\ Institute for Transport Studies, University of Leeds, LS2 \\ 9JT, UK, m.carey@its.leeds.ac.uk \\ and, Ulster Business School, University of Ulster, BT37 \\ 0QB, UK, m.carey@ulster.ac.uk
}

\begin{abstract}
The traffic assignment problem aims to calculate an equilibrium route flow vector, generally by seeking a zero of an appropriate objective function. If a continuous dynamical system follows a descent direction for this objective function at each non-equilibrium route flow vector, the system converges to equilibrium. It is shown that when this dynamical system is discretised with a fixed step length the system eventually approaches close to equilibrium provided that the objective function is continuously differentiable and that the rate of descent is bounded below. Widely used in traffic assignment is the method of successive averages (MSA), which has a decreasing step size at each iteration. With the same conditions as above, it is shown that the resulting dynamical system converges to equilibrium. In the steady state model the necessary conditions are shown to be satisfied provided that the route cost vector is a continuously differentiable monotone function of the route flow vector. In the dynamic queueing model the necessary conditions for convergence are shown not to hold.
\end{abstract}

\section{Introduction}

In steady state traffic models within-day time is not represented, i.e. flows and costs are real-valued (section 2 details a steady state traffic model) whereas in dynamic traffic models, flows and costs are functions of within-day time. This paper focuses primarily on steady state models. The traffic assignment problem is to assign traffic to routes in such a way that no traveller can decrease their travel cost by changing to another route connecting that same origin-destination (OD) pair. Generally this is formulated as a minimisation problem, so that a minimum of the objective function corresponds to user equilibrium. Suppose that a descent vector, say $\phi(\mathbf{X})$, of the objective function can be specified for each route flow vector $\mathbf{X}$. Then by following the curve determined by the direction $\phi(\mathbf{X})$ (which will change as $\mathbf{X}$ changes), the objective function constantly decreases and so the system converges to user equilibrium. In practice in computations, the route flow vector is updated discretely. Often the step length decreases with iteration number in a predetermined way as in the method of successive averages (MSA), which is widely used in traffic assignment (Sheffi, 1985). In order that this discrete system converges, additional conditions must be imposed. Powell and Sheffi (1982) required that the objective function have continuous first and second derivatives (with respect to the route flow vector) and applied this convergence result to the steady state network equilibrium problem. The convergence results in section 4 instead require that the objective function be only once continuously differentiable and that the rate of descent be bounded below by a multiple of $\|\phi(\mathbf{X})\|^{3}$. Firstly, it is shown that for a fixed step length discrete dynamical system, the route flow vector eventually approaches to within a certain distance of equilibrium and furthermore that this distance decreases as the (fixed) step length decreases. Secondly, it is shown that if the sequence of step lengths tends to zero and the series of step lengths is divergent, then the system converges to equilibrium. These convergence results are then shown to hold for the steady state model assuming that the cost on 
each link is a continuously differentiable function of link flow (this assumption does of course limit the applicability of the results). Section 5 shows that continuous differentiability of link cost as a function of link flow does not hold in the dynamic vertical queueing model. Section 6 gives some numerical results to compare MSA with a fixed step length algorithm on several test networks. The convergence of MSA was investigated numerically in a steady state traffic assignment model in Bar-Gera and Boyce (2006). Although MSA is widely used in traffic assignment, the convergence of it can be slow, especially if the initial flow vector is far from equilibrium (this is because the auxiliary flow vector in the descent direction generated at each iteration contributes equally to the final solution). Liu et al. (2009) seek to overcome the slow convergence of MSA by their method of successive weighted averages which uses a step length sequence in which higher weights are given to the auxiliary flow vector generated at the later iterations. Dunn and Harshbarger (1978) showed the convergence of an open loop (i.e. with predetermined step lengths) conditional gradient algorithm. Rather than predetermining the step lengths, the step lengths may be determined at each iteration. Many algorithms choose a step length at each iteration in order to minimise the objective function along the direction of the descent vector, e.g. as in the Frank-Wolfe algorithm (Leblanc et al., 1975). Other methods of adjusting step length to ensure effective convergence include the Armijo-like scheme in Smith (1984b) or self-regulating methods such as in Magnanti and Perakis (1997) or Du et al. (2013).

\section{The steady state traffic assignment model}

The steady state traffic assignment model is the same as that in Smith (1984a). The traffic network is considered to be a directed graph consisting of a set of nodes and a set of links. Suppose that a set $K$ of OD pairs is given and that there is a fixed (positive) demand for travel between each of these OD pairs; let $\rho_{k}$ be the demand for travel between OD pair $k$. A route is defined to be any acyclic path connecting an OD pair. Denote the flow on route $r$ by $X_{r}$ and the route flow vector by $\mathbf{X}=\left(X_{1}, X_{2}, \ldots, X_{N}\right)$ where $N$ is the number of routes in the network. Then the set of feasible route flow vectors, denoted $D$, is given by

$$
D=\left\{X \in \mathbb{R}_{+}^{N}: \sum_{r \in R_{k}} X_{r}=\rho_{k} \forall k \in K\right\}
$$

where $R_{k}$ is the set of all routes joining origin-destination pair $k$. If the route-link incidence matrix $\mathbf{A}$ is defined by

$$
A_{i j}= \begin{cases}1 & \text { if route } j \text { traverses link } i \\ 0 & \text { otherwise }\end{cases}
$$

then the link flow vector, denoted $\mathbf{x}$, can be specified in terms of the route flow vector by $\mathbf{x}=\mathbf{A X}$. For each link $i$, denote by $c_{i}(\mathbf{x})$ the cost on link $i$ when the link flow vector is $\mathbf{x}$. These link cost functions determine the link cost vector $\mathbf{c}(\mathbf{x})=\left(c_{1}(\mathbf{x}), c_{2}(\mathbf{x}), \ldots, c_{n}(\mathbf{x})\right)$ where $n$ is the number of links in the network. Then the route cost vector $\mathbf{C}(\mathbf{X})=\left(C_{1}(\mathbf{X}), C_{2}(\mathbf{X}), \ldots, C_{N}(\mathbf{X})\right)$ can be specified in terms of the link cost vector via the relation

$$
\mathbf{C}(\mathbf{X})=\mathbf{A}^{\mathbf{T}} \mathbf{c}(\mathbf{A X})
$$

Now define $x_{+}^{n}=(\max \{0, x\})^{n}$ for any real $x$ and integer $n$. Then, given a route flow vector $\mathbf{X}$, it is natural to define the day-to-day route swap vector $\phi(\mathbf{X})$ by

$$
\phi(\mathbf{X})=\sum_{k \in K} \sum_{r, s \in R_{k}} X_{r}\left(C_{r}(\mathbf{X})-C_{s}(\mathbf{X})\right)_{+} \delta_{\mathbf{r s}}
$$

where $\delta_{\mathbf{r s}}$ is the swap vector from route $r$ to route $s$, i.e. has -1 in the $r$ th place and 1 in the $s$ th place and zeros elsewhere. Let $\tau$ represent day-to-day time, and view this as a continuous non-negative variable. Then, for $\mathbf{X}_{\mathbf{0}} \in D$, consider the dynamical system

$$
\begin{aligned}
\frac{d \mathbf{X}(\tau)}{d \tau} & =\boldsymbol{\phi}(\mathbf{X})(\tau), \\
\mathbf{X}(0) & =\mathbf{X}_{\mathbf{0}} .
\end{aligned}
$$


By Picard's existence theorem (see e.g. Kreyszig (1978)) the dynamical system (3) has a unique solution trajectory provided that the route swap vector $\phi(\mathbf{X})$ is a Lipschitz continuous function of the route flow vector $\mathbf{X}$. If $\phi(\mathbf{X})$ is defined as in (2) then it is a Lipschitz continuous function of the route flow vector $\mathbf{X}$ provided that the route cost vector $\mathbf{C}(\mathbf{X})$ is a Lipschitz continuous function of $\mathbf{X}$. Clearly equilibrium of this dynamical system occurs when $\phi(\mathbf{X})$ is the zero vector. This is the case if and only if $C_{r}(\mathbf{X})>C_{s}(\mathbf{X})$ implies $X_{r}=0$ for all routes $r$ and $s$ connecting the same OD pair, i.e. for each origin-destination pair more costly routes are not used.

Theorem 1. (Schauder, 1930) Let $E$ be a normed space. Let $K$ be a convex subset of $E$. Let $C$ be a compact, non-empty subset of $K$. Then every continuous map $A: K \rightarrow C$ has at least one fixed point.

Theorem 2. There exists equilibrium of the dynamical system (3).

Proof. Define $T(\mathbf{X})=\mathbf{X}+\alpha \phi(\mathbf{X})$. It is easily shown (Smith and Wisten, 1995) that if $\alpha \leq \frac{1}{X_{\max } C_{\max }}$ where $X_{\max }=\max _{k \in K} \rho_{k}$ is an upper bound for the flows and $C_{\max }$ is an upper bound for the costs, then $T$ maps $D$ to itself. $T$ is a continuous function of $\mathbf{X}$ since $\phi(\mathbf{X})$ is a continuous function of $\mathbf{X}$. Therefore $T$ has at least one fixed point $\mathbf{X}^{*}$ by Theorem 1 . By definition $T\left(\mathbf{X}^{*}\right)=\mathbf{X}^{*}$, or equivalently $\phi\left(\mathbf{X}^{*}\right)=0$, and therefore $\mathbf{X}^{*}$ is an equilibrium of $(3)$.

The dynamical system (3) is globally convergent to equilibrium if, given any starting route flow vector $\mathbf{X}_{\mathbf{0}}$, there is an equilibrium route flow vector $\mathbf{X}^{*}$ such that $\left\|\mathbf{X}(\tau)-\mathbf{X}^{*}\right\| \rightarrow 0$ as $\tau \rightarrow \infty$. Convergence to equilibrium can be shown by specifying a Lyapunov function as in the theorem below, which is a modification of the result in Lyapunov (1907).

Theorem 3. Let $\boldsymbol{\phi}(\mathbf{X})$ be a continuously differentiable function of $\mathbf{X}$. Then the dynamical system (3) is globally convergent to equilibrium if there is a continuously differentiable scalar function $V$, defined throughout D, such that:

(1) $V(\mathbf{X}) \geq 0$ for all $\mathbf{X} \in D$,

(2) $V(\mathbf{X})=0$ if and only if $\mathbf{X}$ is an equilibrium route flow vector, and

(3) $\operatorname{grad} V(\mathbf{X}) \cdot \boldsymbol{\phi}(\mathbf{X})<0$ if $\mathbf{X}$ is not an equilibrium route flow vector.

Smith (1984) showed convergence to equilibrium in the steady state model provided that the cost on each link is a continuously differentiable and non-decreasing function of the flow on that link, by specifying the following Lyapunov function:

$$
V(\mathbf{X})=\sum_{k \in K} \sum_{r, s \in R_{k}} X_{r}\left(C_{r}(\mathbf{X})-C_{s}(\mathbf{X})\right)_{+}^{2} .
$$

\section{Discrete route swap processes}

For practical purposes the traffic assignment problem must be discretised, with the route flow vector at the $(n+1)$ th iteration depending on the route flow vector at the $n$th iteration and the route swap function $\phi$. If the step length $\alpha$ is fixed, we have the following discrete dynamical system:

$$
\mathbf{X}_{\mathbf{n}+\mathbf{1}}=\mathbf{X}_{\mathbf{n}}+\alpha \phi\left(\mathbf{X}_{\mathbf{n}}\right)
$$

In order that the iterates stay within the feasible set, the route swap vector must leave the total flow unchanged for each OD pair (which $\phi(\mathbf{X})$ clearly does) and the value of $\alpha$ must be chosen to be sufficiently small. If the route swap vector is defined as in (2), then

$$
(\mathbf{X}+\alpha \phi(\mathbf{X}))_{r} X_{r}-\alpha \sum_{s: r \sim s} X_{r}\left(C_{r}(\mathbf{X})-C_{s}(\mathbf{X})\right)_{+} \geq X_{r}\left(1-\alpha N C_{\max }\right) \geq 0
$$

provided that $\alpha \leq \frac{1}{N C_{\max }}$ (Smith and Wisten, 1995). 
Figure 1: Counterexample network to descent of MSA

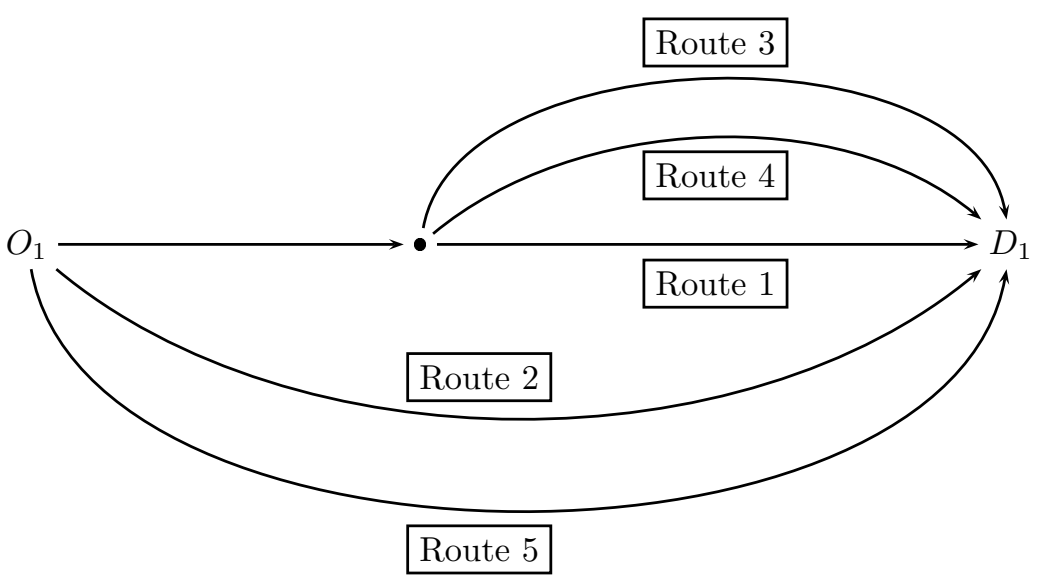

Rather than keeping the step length $\alpha$ fixed, it may be a predetermined function $\alpha_{n}$ of the iteration number $n$, resulting in the discrete dynamical system

$$
\mathbf{X}_{\mathbf{n}+\mathbf{1}}=\mathbf{X}_{\mathbf{n}}+\alpha_{n} \phi\left(\mathbf{X}_{\mathbf{n}}\right)
$$

where $\alpha_{n}>0$ for all $n$. Of course $\alpha_{0}$ must be chosen small enough so that the iterates stay within the feasible set $D$ as discussed above. When the sequence of predetermined step lengths is chosen to be $\alpha_{n}=\frac{1}{n}$, this is called the method of successive averages (MSA), because the vector at each iteration can be expressed as the average of a sequence of vectors along the descent direction over the preceding iterations(Sheffi, 1985). Powell and Sheffi (1982) showed that if the objective function is twice continuously differentiable and descent of the objective function is guaranteed for all non-equilibrium $\mathbf{X}$, then (5) converges to equilibrium provided that (20) holds and

$$
\sum_{n=1}^{\infty}\left(\alpha_{n}\right)^{2}<\infty
$$

A commonly used swap vector with MSA is the all or nothing assignment to the least costly route (or routes), i.e.

$$
(\phi(\mathbf{X}))_{r}= \begin{cases}-\frac{T_{k}}{n} & \text { if } r \notin k_{\min }(\mathbf{X}), \\ \frac{\left|R_{k}\right|-\mid k_{\min }(\mathbf{X})}{\mid k_{\min }(\mathbf{X} \mid} T_{k} & \text { if } r \in k_{\min }(\mathbf{X}),\end{cases}
$$

where route $r$ connects OD pair $k, T_{k}$ is the total flow between OD pair $k$ and $k_{\min }(\mathbf{X})$ is the set of least costly routes connecting OD pair $k$. If there is only one least costly route at $\mathbf{X}$, then the route swap vector $\phi(\mathbf{X})$ defined in (7) is a descent direction (Bernstein, 1990, Mounce and Carey, 2010) for the objective function

$$
V(\mathbf{X})=\sum_{k \in K} \sum_{r \in R_{k}} X_{r}\left(C_{r}(\mathbf{X})-C_{k_{m i n}}(\mathbf{X})\right)_{+}^{2} .
$$

However, if there is more than one least costly route at $\mathbf{X}$, then $\boldsymbol{\phi}$ defined in (7) may not be a descent direction for (8). To see this, consider the network in Figure 1 with five routes connecting one OD pair. Suppose that $C_{1}(\mathbf{X})=C_{2}(\mathbf{X})=1, C_{3}(\mathbf{X})=C_{4}(\mathbf{X})=1.1$ and $C_{5}(\mathbf{X})=2$. Then the swap vector $\phi(\mathbf{X})=(1.5,1.5,-1,-1,-1)^{T}$, the link cost derivative $x_{1}^{\prime}=-0.5$ and the objective function

$$
\begin{aligned}
V(\mathbf{X}) & \left.=X_{3}\left(C_{3}(\mathbf{X})-C_{1}(\mathbf{X})\right)+X_{4}\left(C_{4}(\mathbf{X})\right)-C_{1}(\mathbf{X})\right)+X_{5}\left(C_{5}(\mathbf{X})-C_{1}(\mathbf{X})\right) \\
& =\frac{X_{3}}{100}+\frac{X_{3}}{100}+X_{5} .
\end{aligned}
$$


Suppose that all link cost functions have zero derivative apart from link 1 . Then

$$
\begin{aligned}
V^{\prime}(\mathbf{X} ; \phi(\mathbf{X}))= & X_{3}^{\prime}\left(C_{3}(\mathbf{X})-C_{1}(\mathbf{X})\right)+X_{4}^{\prime}\left(C_{4}(\mathbf{X})-C_{1}(\mathbf{X})+X_{5}^{\prime}\left(C_{5}(\mathbf{X})-C_{1}(\mathbf{X})\right)\right. \\
& +X_{5}\left(C_{5}(\mathbf{X})-C_{1}(\mathbf{X})\right)\left(C_{5}^{\prime}(\mathbf{X})-C_{1}^{\prime}(\mathbf{X})\right) \\
= & \frac{X_{3}^{\prime}+X_{4}^{\prime}}{100}+X_{5}^{\prime}-X_{5} x_{1}^{\prime} c_{1}^{\prime}(\mathbf{x}) \\
= & -\frac{51}{50}+\frac{X_{5}}{2} c_{1}^{\prime}(\mathbf{x}) \\
> & 0
\end{aligned}
$$

if $c_{1}(\mathbf{x})$ is sufficiently large. Hence $\phi$ defined in (7) is not a descent direction for (8) in this case. In practice, the likelihood of having two routes of equal cost is probably quite small. However, if two or more routes have very similar costs, descent may hold only along a vector of small magnitude.

\section{Convergence of the discrete route swap processes}

In this section the convergence of the discrete route swap processes detailed above is considered. The following lemmas will be required.

Lemma 1. In the steady state model, the route cost vector $\mathbf{C}(\mathbf{X})$ is a continuously differentiable function of $\mathbf{X}$ provided that each link cost function $c_{i}(\mathbf{x})$ is a continuously differentiable function of $\mathbf{x}$.

Proof. Differentiating (1) with respect to $\mathbf{X}$ gives $\mathbf{C}^{\prime}(\mathbf{X})=\mathbf{A}^{\mathbf{T}} \mathbf{c}^{\prime}(\mathbf{A X}) \mathbf{A}$ and the result then follows immediately.

This implies that both $V(\mathbf{X})$ and $\phi(\mathbf{X})$ are continuously differentiable functions of $\mathbf{X}$ provided that each link cost function is continuously differentiable. In this case $\phi(\mathbf{X})$ is a continuously differentiable function of $\mathbf{X}$ defined on the compact set $D$ and is therefore a Lipschitz function of $\mathbf{X}$. By Picard's theorem this is sufficient for the existence of a solution trajectory of (3) (see e.g. Kreyszig, 1978).

Definition 1. The route cost vector is a monotone function of the route flow vector if and only if

$$
(\mathbf{c}(\mathbf{x})-\mathbf{c}(\mathbf{y})) \cdot(\mathbf{x}-\mathbf{y}) \geq 0
$$

for all route flow vectors $\mathbf{x}$ and $\mathbf{y}$.

Monotonicity of the route cost vector certainly holds if the cost on each link is a nondecreasing function of the flow on that link.

Lemma 2. If the route cost vector is a monotone function of the route flow vector and the route swap vector is as defined in (2), then for some constant $\beta>0$,

$$
\operatorname{grad} V(\mathbf{X}) \cdot \phi(\mathbf{X}) \leq-\beta\|\phi(\mathbf{X})\|^{3}
$$

holds for all $\mathbf{X}$.

Proof. Assuming that route cost is a monotone function of route flow, it is easily shown (Smith, 1984) that

$$
\operatorname{grad} V(\mathbf{X}) \cdot \phi(\mathbf{X}) \leq-\sum_{k \in K} \sum_{r, s \in R_{k}} X_{r}\left(C_{r}(\mathbf{X})-C_{s}(\mathbf{X})\right)_{+}^{3}
$$

Then define $u$ and $v$ to be the choice of $r$ and $s$ (respectively) that maximises $X_{r}\left(C_{r}(\mathbf{X})-C_{s}(\mathbf{X})\right)_{+}$. Also define $B=\max _{k \in K}\left|R_{k}\right|$. Since

$$
\|\phi(\mathbf{X})\| \leq B X_{u}\left(C_{u}(\mathbf{X})-C_{v}(\mathbf{X})\right)_{+}
$$


and $X_{r} \leq X_{\max }$, it follows that

$$
\begin{aligned}
\sum_{k \in K} \sum_{r, s \in R_{k}} X_{r}\left(C_{r}(\mathbf{X})-C_{s}(\mathbf{X})\right)_{+}^{3} & \geq X_{u}\left(C_{u}(\mathbf{X})-C_{v}(\mathbf{X})\right)_{+}^{3} \\
& \geq \frac{\left[X_{u}\left(C_{u}(\mathbf{X})-C_{v}(\mathbf{X})\right)_{+}\right]^{3}}{\left(X_{\max }\right)^{2}} \\
& \geq \frac{\|\phi(\mathbf{X})\|^{3}}{B^{3}\left(X_{\max }\right)^{2}}
\end{aligned}
$$

Notice that $\phi(\mathbf{X})$ is a convergence measure since it is a non-negative function that is zero only at equilibrium (since if $\|\phi(\mathbf{X})\|=0$ no swapping occurs because there is no incentive to change route).

Lemma 3. The objective function $V(\mathbf{X})=\sum_{k \in K} \sum_{r, s \in R_{k}} X_{r}\left(C_{r}(\mathbf{X})-C_{s}(\mathbf{X})\right)_{+}^{2}$ converges to zero if and only if the route swap vector $\boldsymbol{\phi}(\mathbf{X})$ (as defined in (2)) converges to zero.

Proof. Defining $u$ and $v$ as in Lemma 2,

$$
\begin{aligned}
V(\mathbf{X})=\sum_{k \in K} \sum_{r, s \in R_{k}} X_{r}\left(C_{r}(\mathbf{X})-C_{s}(\mathbf{X})\right)_{+}^{2} & \geq X_{u}\left(C_{u}(\mathbf{X})-C_{v}(\mathbf{X})\right)_{+}^{2} \\
& \geq \frac{\left(X_{u}\left(C_{u}(\mathbf{X})-C_{v}(\mathbf{X})\right)_{+}\right)^{2}}{M} \\
& \geq \frac{\|\phi(\mathbf{X})\|^{2}}{M|K|^{2}\left(R_{\max }\right)^{2}} .
\end{aligned}
$$

Therefore

$$
\|\phi(\mathbf{X})\| \leq \sqrt{B^{2} M V(\mathbf{X})}
$$

and then clearly $\|\phi(\mathbf{X})\| \rightarrow 0$ as $V(\mathbf{X}) \rightarrow 0$.

Now we show the converse. Firstly, define $S_{k}=\left\{r \in R_{k}: r \neq u, v\right.$ and $\left.C_{r}(\mathbf{X}) \geq C_{u}(\mathbf{X})\right\}$. Then notice that if $X_{r}\left(C_{r}(\mathbf{X})-C_{u}(\mathbf{X})\right)_{+} \geq 0$ and $X_{u}\left(C_{u}(\mathbf{X})-C_{v}(\mathbf{X})\right)_{+} \geq 0$ then

$$
X_{r}\left(C_{r}(\mathbf{X})-C_{v}(\mathbf{X})\right)_{+} \geq X_{r}\left(C_{r}(\mathbf{X})-C_{u}(\mathbf{X})\right)_{+} .
$$

Therefore

$$
\sum_{r \in S_{k}} X_{r}\left(C_{r}(\mathbf{X})-C_{v}(\mathbf{X})\right)_{+} \geq \sum_{r \in S_{k}} X_{r}\left(C_{r}(\mathbf{X})-C_{u}(\mathbf{X})\right)_{+}
$$

If either

$$
\sum_{r \in R_{k}: r \neq u, v} X_{r}\left(C_{r}(\mathbf{X})-C_{u}(\mathbf{X})\right)_{+} \leq 0
$$

or

$$
\sum_{r \in R_{k}: r \neq u, v} X_{r}\left(C_{r}(\mathbf{X})-C_{v}(\mathbf{X})\right)_{+} \geq 0
$$

then clearly

$$
\|\phi(\mathbf{X})\| \geq X_{u}\left(C_{u}(\mathbf{X})-C_{v}(\mathbf{X})\right)_{+} .
$$

However, if we assume that both (12) and (13) do not hold, then we can deduce that

$$
\sum_{r \in S_{k}} X_{r}\left(C_{r}(\mathbf{X})-C_{v}(\mathbf{X})\right)_{+}<\sum_{r \in S_{k}} X_{r}\left(C_{r}(\mathbf{X})-C_{u}(\mathbf{X})\right)_{+} .
$$

In (15), the right hand side must be zero and the left hand side must be nonnegative, which is a contradiction. Therefore at least one of (12) or (13) must hold, in which case (14) holds. Hence

$$
V(\mathbf{X}) \leq|K| B X_{u}\left(C_{u}(\mathbf{X})-C_{v}(\mathbf{X})\right)_{+} \leq|K| B\|\boldsymbol{\phi}(\mathbf{X})\| .
$$

Therefore $V(\mathbf{X}) \rightarrow 0$ as $\|\phi(\mathbf{X})\| \rightarrow 0$. 
The following theorem shows that if $\alpha$ is chosen small enough, then the dynamical system (4) converges to within a small distance of equilibrium (and may continue converging).

Theorem 4. Suppose that $V(\mathbf{X})$ is a Lyapunov function for the continuous dynamical system (3) and that $\boldsymbol{\phi}(\mathbf{X})$ is a continuous function of $\mathbf{X}$. Suppose also that $V(\mathbf{X})$ is a continuously differentiable function of $\mathbf{X}$ with (10) holding for all route flow vectors $\mathbf{X}$ for some (common) constant $\beta$. Then the discrete dynamical system (4) converges to within a certain distance of equilibrium, which decreases as $\alpha$ decreases.

Proof. Let $\gamma$ be the line segment joining $\mathbf{X}_{\mathbf{n}}$ and $\mathbf{X}_{\mathbf{n}+\mathbf{1}}$, i.e.

$$
\gamma(u)=\mathbf{X}_{\mathbf{n}}+u \alpha \phi\left(\mathbf{X}_{\mathbf{n}}\right)
$$

for $u \in[0,1]$. Let $\phi_{\max }$ be a bound for $\phi(\mathbf{X})$ on the feasible set $D$ (which exists since $\boldsymbol{\phi}(\mathbf{X})$ is continuous on the compact set $D$ ). Since $V$ is continuously differentiable on the compact set $D$, given any $\epsilon>0$ it is possible to choose $\alpha$ small enough so that if $\|\mathbf{X}-\mathbf{Y}\| \leq \alpha \phi_{\max }$ then

$$
\|\nabla V(\mathbf{X})-\nabla V(\mathbf{Y})\|<\epsilon .
$$

Certainly this is satisfied on the line segment $\gamma$ and therefore

$$
\begin{aligned}
V\left(\mathbf{X}_{\mathbf{n}+\mathbf{1}}\right)-V\left(\mathbf{X}_{\mathbf{n}}\right) & =\int_{0}^{1}\left(\nabla V\left(\mathbf{X}_{\mathbf{n}}+u \alpha \phi\left(\mathbf{X}_{\mathbf{n}}\right)\right)\right)\left(\alpha \phi\left(\mathbf{X}_{\mathbf{n}}\right)\right) d u \\
& =\int_{0}^{1}\left(\nabla V\left(\mathbf{X}_{\mathbf{n}}+u \alpha \phi\left(\mathbf{X}_{\mathbf{n}}\right)\right)-\nabla V\left(\mathbf{X}_{\mathbf{n}}\right)\right)\left(\alpha \phi\left(\mathbf{X}_{\mathbf{n}}\right)\right) d u+\int_{0}^{1}\left(\nabla V\left(\mathbf{X}_{\mathbf{n}}\right)\right)\left(\alpha \boldsymbol{\phi}\left(\mathbf{X}_{\mathbf{n}}\right)\right) d u \\
& \leq \epsilon \alpha\left\|\boldsymbol{\phi}\left(\mathbf{X}_{\mathbf{n}}\right)\right\|-\beta \alpha\left\|\phi\left(\mathbf{X}_{\mathbf{n}}\right)\right\|^{3} \\
& =\left\|\phi\left(\mathbf{X}_{\mathbf{n}}\right)\right\|\left(\epsilon \alpha-\beta \alpha\left\|\phi\left(\mathbf{X}_{\mathbf{n}}\right)\right\|^{2}\right)
\end{aligned}
$$

where $\beta$ is the positive constant satisfying (10). For a given $\phi_{\min }>0$ it is certainly possible to choose $\alpha$ small enough so that 16 is satisfied with

$$
\epsilon<\frac{\beta}{2}\left(\phi_{\min }\right)^{2}
$$

in which case

$$
V\left(\mathbf{X}_{\mathbf{n}+\mathbf{1}}\right)-V\left(\mathbf{X}_{\mathbf{n}}\right)<-\frac{\beta \alpha}{2}\|\phi(\mathbf{X})\|^{3}<-\frac{\beta \alpha}{2}\left(\phi_{\min }\right)^{3}
$$

provided $\left\|\phi\left(\mathbf{X}_{\mathbf{n}}\right)\right\| \geq \phi_{\min }$.

While the sequence of route flow vectors stays in the set $\left\{\mathbf{X} \in D:\|\phi(\mathbf{X})\| \geq \phi_{\min }\right\}$, (17) is satisifed at each iteration and therefore

$$
V\left(\mathbf{X}_{\mathbf{n}}\right)-V\left(\mathbf{X}_{\mathbf{0}}\right)<-n \frac{\beta \alpha}{2}\left(\phi_{\min }\right)^{3}
$$

Consequently, after a sufficient number of iterations the sequence must eventually reach a point $\mathbf{X}_{\mathbf{n}}$ with $\left\|\phi\left(\mathbf{X}_{\mathbf{n}}\right)\right\|<\phi_{\min }$. Also, since $\boldsymbol{\phi}(\mathbf{X})$ is a continuous function of $\mathbf{X}$, given any $\epsilon>0$, it is possible to choose $\alpha$ small enough so that

$$
\left\|\phi\left(\mathbf{X}_{\mathbf{n}+\mathbf{1}}\right)-\phi\left(\mathbf{X}_{\mathbf{n}}\right)\right\|<\epsilon
$$

and then

$$
\left\|\phi\left(\mathbf{X}_{\mathbf{n}+\mathbf{1}}\right)\right\| \leq\left\|\phi\left(\mathbf{X}_{\mathbf{n}}\right)\right\|+\left\|\phi\left(\mathbf{X}_{\mathbf{n}+\mathbf{1}}\right)-\phi\left(\mathbf{X}_{\mathbf{n}}\right)\right\|<\phi_{\min }+\epsilon .
$$

By choosing $\alpha$ small enough so that (18) is satisfied with $\epsilon=\phi_{\min }$, further elements of the sequence remain in the set $\left\{\mathbf{X} \in D:\|\phi(\mathbf{X})\|<2 \phi_{\min }\right\}$.

Notice that Theorem 4 requires that the route cost vector is a continuously differentiable function of the route flow vector. In the steady state model, this is the case provided that the cost on each link is a continuously differentiable function of the link flow. In the dynamic model, this may not hold. In section 5 it will be shown that in the dynamic queueing model there is a discontinuity in the cost gradient when the inflow rate is equal to capacity. 
In Theorem 4, the smaller the choice of $\alpha$, the closer the dynamical system (4) eventually gets to equilibrium. Although a descent direction can be specified after this point, the step length may then be too large to decrease the objective function. In this case, a way around this is to periodically decrease $\alpha$ as the system reaches a series of thresholds. Another method of ensuring that convergence to equilibrium continues even when the system is close to equilibrium is to decrease $\alpha$ at each iteration in a predetermined way as in the method of successive averages.

Dunn (1976) showed that, in a Hilbert space, for functions with critical points (i.e. a point where the function is not differentiable or has zero derivative) the gradient descent algorithm

$$
x_{n+1}=x_{n}-\alpha_{n} \nabla f\left(x_{n}\right)
$$

converges (at least weakly) to some critical point of $f$ (where $f$ necessarily attains its global minimum) from an arbitrary starting point $x_{0}$ provided that

and

$$
\lim _{n \rightarrow \infty} \alpha_{n}=0
$$

$$
\sum_{n=0}^{\infty} \alpha_{n}=\infty .
$$

In the following theorem a similar result is shown, but for a general (rather than a gradient) descent direction.

Theorem 5. Suppose that $V(\mathbf{X})$ is a Lyapunov function for the continuous dynamical system (3) and that $\phi(\mathbf{X})$ is a continuous function of $\mathbf{X}$. Suppose also that $V(\mathbf{X})$ is a continuously differentiable function of $\mathbf{X}$ with (10) holding for all route flow vectors $\mathbf{X}$ for some (common) constant $\beta$. Suppose also that the step length sequence $\left(\alpha_{n}\right)_{n=0}^{\infty}$ satisfies (19) and (20). Then the discrete dynamical system (5) is guaranteed to converge to equilibrium.

Proof. Given any $\phi_{\min }$, from Theorem 4 it is possible to choose $\alpha$ small enough so that (17) holds for all $\mathbf{X} \in D$ with $\|\phi(\mathbf{X})\| \geq \phi_{\min }$. Let $\alpha_{m}$ be the first member of the sequence $\left(\alpha_{n}\right)_{n=1}^{\infty}$ such that $(17)$ holds. Then for all $n>m$,

$$
V\left(\mathbf{X}_{\mathbf{n}}\right)-V\left(\mathbf{X}_{\mathbf{m}}\right)<-\frac{\beta}{2}\left(\phi_{\min }\right)^{3} \sum_{i=m+1}^{n} \alpha_{i}
$$

and therefore

$$
V\left(\mathbf{X}_{\mathbf{n}}\right)<V\left(\mathbf{X}_{\mathbf{m}}\right)-\frac{\beta}{2}\left(\phi_{\min }\right)^{3} \sum_{i=m+1}^{n} \alpha_{i}
$$

provided that $\mathbf{X}_{\mathbf{m}}, \mathbf{X}_{\mathbf{m}+\mathbf{1}}, \ldots \mathbf{X}_{\mathbf{n}-\mathbf{1}}$ all have norm at least equal to $\phi_{\min }$. Since (20) holds, the sequence on the right hand side of $(21)$ is unbounded with $n$. Therefore, eventually a point $\mathbf{X}_{\mathbf{n}}$ must be reached with $\left\|\phi\left(\mathbf{X}_{\mathbf{n}}\right)\right\|<\phi_{\min }$. Also, by continuity of $\boldsymbol{\phi}$ (with respect to $\mathbf{X}$ ), $\alpha(n)$ can be chosen small enough (i.e. by choosing $n$ large enough) so that $\left\|\phi\left(\mathbf{X}_{\mathbf{n}+\mathbf{1}}\right)\right\|<2 \phi_{\min }$, in which case all future iterations will certainly satisfy $\|\phi(\mathbf{X})\|<2 \phi_{\min }$. Since $\phi_{\min }$ was arbitrary, it is clear that $\left\|\phi\left(\mathbf{X}_{\mathbf{n}}\right)\right\| \rightarrow 0$ as $n \rightarrow \infty$. Since $\left\|\phi\left(\mathbf{X}_{\mathbf{n}}\right)\right\|=0$ if and only if $\mathbf{X}$ is at equilibrium, it is clear that the dynamical system (5) converges to equilibrium as $n \rightarrow \infty$.

Note that in Theorems 4 and $5, V$ is a Lyapunov function by hypothesis and so continually decreases with day-to-day time. However, in the discrete dynamical system not every step is guaranteed to reduce $V$; this depends on the step size. However, (19) ensures that the step lengths eventually become small enough to ensure descent and (20) ensures that convergence continues to occur even after a large number of iterations.

Notice that Theorem 5 has weaker hypotheses than the result in Sheffi and Powell (1985) in that it does not require that the second derivative of the objective function even exists. It is also weaker since it requires (6) to be satisfied as well as (19). However, Theorem 5 has a stronger condition on the reduction in the objective function in the direction of the descent vector. 


\section{Discontinuity of the cost derivative in the dynamic vertical queueing model}

The following theorem shows that in the dynamic vertical queueing model (Smith and Wisten, 1995, Mounce, 2006), the cost derivative is not continuous (where the cost derivative is the directional liminf derivative as used in Mounce (2006)). Therefore the convergence results in section 4 cannot be applied to that model. For more on discretisation of the dynamical model refer to Mounce and Carey (2011).

Theorem 6. The directional derivative $\delta \mathbf{C}(\mathbf{X} ; \phi$ is a discontinuous function of the route flow vector $\mathbf{X}$ in the dynamic vertical queueing model.

Proof. Consider a simple two link network with route 1 consisting of a single bottleneck link with capacity 1 and route 2 consisting of a single link with fixed cost. Let $\mathbf{X}$ be the route flow vector with $X_{1}=1$ and $X_{2}=0$ for all $t \in[0,1]$. Let $\mathbf{Y}$ be the route flow vector with $Y_{1}=1-\epsilon$ and $Y_{2}=\epsilon$ for all $t \in[0,1]$. Also, let $\phi$ be the route swap vector with $\phi_{1}=1$ and $\phi_{2}=-1$ for all $t \in[0,1]$. Then for any $t \in[0,1], \delta \mathbf{C}(\mathbf{X} ; \phi)(t)=(t, 0)$ whereas $\delta \mathbf{C}(\mathbf{Y} ; \boldsymbol{\phi})(t)=(0,0)$. Therefore $\|\mathbf{X}-\mathbf{Y}\|=\epsilon$ but $\|\delta \mathbf{C}(\mathbf{X} ; \boldsymbol{\phi})-\delta \mathbf{C}(\mathbf{Y} ; \boldsymbol{\phi})\|=1$ no matter how small $\epsilon$ is.

Theorems 4 and 5 require that the objective function $V(\mathbf{X})$ be a continuously differentiable function of the route flow vector $\mathbf{X}$. Theorem 6 shows that this is not satisifed in the dynamic queueing model (and it is possibly not in other dynamic models). Hence Theorems 4 and 5 cannot be applied. Theorems 4 and 5 also require that $V$ be a Lyapunov function for the dynamical system (3). Mounce (2006) shows that $V(\mathbf{X})=\sum_{k \in K} \sum_{r, s \in R_{k}} \int_{0}^{1} X_{r}(t)\left(C_{r}^{X}(t)-C_{s}^{X}(t)\right)_{+}^{2} d t$ is a Lyapunov function for $(3)$ when $\mathbf{C}(\mathbf{X})$ is a monotone function of $\mathbf{X}$, i.e. if and only if

$$
(\mathbf{X}-\mathbf{Y}) \cdot(\mathbf{C}(\mathbf{X})-\mathbf{C}(\mathbf{Y}))=\sum_{r} \int_{0}^{1}\left(C_{r}^{X}(t)-C_{r}^{Y}(t)\right)\left(X_{r}(t)-Y_{r}(t)\right) d t \geq 0
$$

for all route flow vectors $\mathbf{X}$ and $\mathbf{Y}$. However, the route cost vector is not in general a monotone function of the route flow vector in the dynamic vertical queueing model (Mounce and Smith, 2007). A Lyapunov function is not known for general networks. Both of these facts are barriers to the application of Theorems 4 and 5 to the dynamic model. However, it is important to note that Theorem 6 does not show that MSA does not converge in the dynamic model.

\section{Numerical results}

In this section, numerical results are given for a route based traffic assignment model (programmed in $\mathrm{C} \#$ and run on a $2.8 \mathrm{GHz}$ Intel i7 with $4 \mathrm{~Gb}$ RAM) run on the following networks:

(1) A simple network with 6 nodes, 9 links and 1 OD pair.

(2) The Sioux Falls network (supplied by Hai Yang and Meng Qiang) with 24 nodes, 76 links and 528 OD pairs.

(3) The Austin network (supplied by Chie Xie) with 7388 nodes, 18961 links and 8487 OD pairs (reduced from the original 649859 OD pairs).

The objective function used for measuring convergence in the results is the standard relative gap given by

$$
V(\mathbf{X})=\sum_{k \in K} \sum_{r \in R_{k}} X_{r}\left(C_{r}(\mathbf{X})-C_{k_{\min }}(\mathbf{X})\right)_{+}
$$

(for more on different convergence measures refer to Mounce and Carey (2010, 2011)). The route swapping algorithm is summarised in Figure 6. Dijkstra's algorithm (Dijkstra, 1959) with binary heaps (Williams, 1964) was used to calculate shortest paths. At the first iteration all traffic was loaded onto the least costly route(s) for each OD pair. For MSA with swapping to the least cost routes the initial step length was chosen to be 0.5 , which is standard. For fixed step length with pairwise swapping, the initial step length was chosen to be the reciprocal of the maximum cost over all routes at the first iteration. For MSA with pairwise swapping the initial step length was chosen to be 0.5 . This did mean that descent did 
Figure 2: Traffic assignment algorithm

\section{Initialisation.}

Use Dijkstra's algorithm to find the least costly route(s) for each OD pair.

Assign all traffic onto shortest paths for each OD pair.

Set initial step length.

\section{Route flow reassignment.}

Adjust step length if using MSA (to initial step length divided by iteration number).

Swap route flows (either pairwise swapping or towards the least costly route).

\section{Gap calculation.}

Calculate new link and then route costs.

Use Dijkstra's algorithm to calculate new shortest routes for all OD pairs.

Calculate equilibrium gap. Is the gap less than or equal to the threshold?

Yes

\section{Terminate}

Figure 3: Results for the simple network

\begin{tabular}{|c|c|c|c|c|c|c|}
\hline Descent vector & \multicolumn{2}{|c|}{ pairwise swap in (2) } & \multicolumn{2}{c|}{ pairwise swap in (2) } & swap to least costly route in (7) \\
\hline Step length & \multicolumn{2}{|c|}{ fixed } & \multicolumn{2}{c|}{$\alpha_{n}=\frac{1}{n}$} & \multicolumn{2}{c|}{$\alpha_{n}=\frac{1}{n}$} \\
\hline Initial step length & \multicolumn{2}{c|}{0.00472} & \multicolumn{2}{c|}{0.5} & \multicolumn{2}{c|}{0.5} \\
\hline Relative equilibrium gap & $1 \%$ & $0.1 \%$ & $1 \%$ & $0.1 \%$ & $1 \%$ & $0.1 \%$ \\
\hline Number of iterations & 36 & 89 & 133 & 221 & 84 & 469 \\
\hline Computation time & $0.3 \mathrm{~s}$ & $0.74 \mathrm{~s}$ & $1.17 \mathrm{~s}$ & $1.94 \mathrm{~s}$ & $0.97 \mathrm{~s}$ & $5.39 \mathrm{~s}$ \\
\hline
\end{tabular}

Figure 4: Results for the Sioux Falls network

\begin{tabular}{|c|c|c|c|c|c|c|}
\hline Descent vector & \multicolumn{2}{|c|}{ pairwise swap in (2) } & \multicolumn{2}{c|}{ pairwise swap in (2) } & swap to least costly route in (7) \\
\hline Step length & \multicolumn{2}{|c|}{ fixed } & \multicolumn{2}{c|}{$\alpha_{n}=\frac{1}{n}$} & \multicolumn{2}{c|}{$\alpha_{n}=\frac{1}{n}$} \\
\hline Initial step length & \multicolumn{2}{|c|}{0.00046} & \multicolumn{2}{c|}{0.5} & \multicolumn{2}{c|}{0.5} \\
\hline Relative equilibrium gap & $1 \%$ & $0.1 \%$ & $1 \%$ & $0.1 \%$ & $1 \%$ & $0.1 \%$ \\
\hline Number of iterations & 422 & 1858 & 4436 & 18864 & 19 & 108 \\
\hline Computation time & $10.6 \mathrm{~s}$ & $46.7 \mathrm{~s}$ & $4 \mathrm{~m} 43 \mathrm{~s}$ & $25 \mathrm{~m} 16 \mathrm{~s}$ & $0.5 \mathrm{~s}$ & $2.7 \mathrm{~s}$ \\
\hline
\end{tabular}

Figure 5: Results for the Austin network

\begin{tabular}{|c|c|c|c|c|c|c|}
\hline Descent vector & \multicolumn{2}{|c|}{ pairwise swap in (2) } & pairwise swap in (2) & swap to least costly route in (7) \\
\hline Step length & \multicolumn{2}{|c|}{ fixed } & \multicolumn{2}{c|}{$\alpha_{n}=\frac{1}{n}$} & \multicolumn{2}{c|}{$\alpha_{n}=\frac{1}{n}$} \\
\hline Initial step length & \multicolumn{2}{c|}{0.00157} & \multicolumn{2}{c|}{0.5} & \multicolumn{2}{c|}{0.5} \\
\hline Relative equilibrium gap & $1 \%$ & $0.1 \%$ & $1 \%$ & $0.1 \%$ & $1 \%$ & $0.1 \%$ \\
\hline Number of iterations & 45 & 839 & N/A & 15 & 69 \\
\hline Computation time & $11 \mathrm{~m} 54 \mathrm{~s}$ & $7 \mathrm{~h} 35 \mathrm{~m} 12 \mathrm{~s}$ & \multicolumn{2}{c|}{ N/A } & $4 \mathrm{~m} 22 \mathrm{~s}$ & $21 \mathrm{~m} 41 \mathrm{~s}$ \\
\hline
\end{tabular}


not occur until the step length had decreased somewhat (for the simple network this took 80 iterations and $0.7 \mathrm{~s}$, whereas for the Sioux Falls network this took 2250 iterations and 2m09s. Interestingly, at least for the Sioux Falls network this is approximately equal to the initial step length calculated for pairwise swapping). Firstly, notice that MSA with pairwise swapping performed poorly for all the networks (it was not applied to the Austin network since it would take too long to converge). For the simple network, pairwise swapping with fixed step length outperformed MSA with swapping to the least costly route, whereas on the larger Sioux Falls and Austin networks the reverse is true. In summary, it is clear that MSA works well on large networks when combined with a swap towards the least costly route(s). It should be noted that for large networks a bush-based algorithm (Dial, 2006, Nie, 2010) is much more efficient for calculating equilibrium with precision.

\section{Conclusion}

The paper addressed the issue of discretising a convergent continuous dynamical system within the context of traffic assignment. It was shown that, provided the objective function is continuously differentiable and the rate of descent satisifies a lower bound inequality, the discrete dynamical system eventually approaches close to equilibrium; furthermore this distance from equilibrium decreases as the (fixed) step length decreases. When the step size decreases with iteration number, the resulting discrete dynamical system is shown to converge to equilibrium. The necessary conditions were shown to hold in the steady state model, but not to hold in the dynamic vertical queueing model. It remains to be shown whether these conditions are satisfied in other dynamic models.

\section{Acknowledgements}

This research was supported by the Engineering and Physical Sciences Research Council under grant $\mathrm{EP} / \mathrm{COO} 7913 / 1$.

\section{References}

Bar-Gera, H. and Boyce, D. 2006. Solving a non-convex combined travel forecasting model by the method of successive averages with constant step sizes, Transportation Research Part $B$ 40, 351-367.

Bernstein, D.H. 1990. Programmability of Continuous and Discrete Network Equilibria, Ph. D. Thesis, University of Illinois at Chicago, USA.

Dial, R.B. 2006. A path-based user-equilibrium traffic assignment algorithm that obviates path storage and enumeration, Transportation Research Part B 40, 917-936.

Dijkstra, E.W. 1959. A note on two problems in connexion with graphs. Numerische Mathematik 1, 269-271.

Du, J., Wong, S.C., Shu, C., Xiong, T., Zhang, M. and Choi, K. 2013. Revisiting Jiang's dynamic continuum model for urban cities, Transportation Research Part B 56, 96-119.

Dunn, J.C. 1976. Convexity, monotonicity, and gradient processes in Hilbert space, Journal of Mathematical Analysis and Applications 53, 145-158.

Dunn, J.C. and Harshbarger, S. 1978. Conditional gradient algorithms with open loop step size rules, Journal of Mathematical Analysis and Applications 62, 432-444.

Kreyszig, E. 1978. Introductory Functional Analysis with applications, John Wiley and Sons Inc, USA.

LeBlanc, L.J., Morlok, E.K. and Pierskalla, W. 1975. An efficient approach to solving the road network equilibrium traffic assignment problem, Transportation Research Part B 9, 309-318.

Liu, H.X., He, X. and He, B. 2009. Method of successive weighted averages (MSWA) and self-regulated averaging schemes for solving stochastic user equilibrium problem, Networks and Spatial Economics 9, 485-503. 
Lyapunov, A.M. 1907. Probleme general de la stabilite du mouvement, Ann. Fac. Sci. Univ. Toulouse (2) 9, 203-474. (French translation of the original paper published in 1892 in Comm. Soc. Math. Kharkow and reprinted in 1947 as Ann. Math. Studies 17, Princeton Univ. Press, Princeton, N.J.)

Magnanti, T.L. and Perakis, G. 1997. Averaging schemes for variational inequalities and systems of equations, Mathematics of Operations Research 22, 568-587.

Mounce, R. 2006. Convergence in a continuous dynamic queueing model for traffic networks, Transportation Research Part B 40, 779-791.

Mounce, R. and Smith, M. 2007. Uniqueness of equilibrium in steady state and dynamic traffic networks. Allsop, R.E. Bell, M.G.H. and Heydecker, B.G., eds. Transportation and Traffic Theory, Elsevier, 281-299.

Mounce, R. and Carey, M. 2010. Route swap processes and convergence measures in dynamic traffic assignment. Tampére C.M.J., Viti F. and Immers L.H., eds. New Developments in Transport Planning: Advances in Dynamic Traffic Assignment, Edward Elgar, 107-130.

Mounce, R. and Carey, M. 2011. Route swapping in dynamic traffic networks, Transportation Research Part B 45, 102-111.

Nie, Y. 2010. A class of bush-based algorithms for the traffic assignment problem, Transportation Research Part B 44, 73-89.

Powell, W. and Sheffi, Y. 1982. The convergence of equilibrium algorithms with predetermined step sizes, Transportation Science 16, 45-55.

Schauder, J. 1930. Der Fixpunktsatz in Funktionalräumen, Studia Mathematica 2, 171-180.

Sheffi, Y. 1985. Urban Transportation Networks: Equilibrium Analysis with Mathematical Programming Methods, Prentice-Hall, USA.

Smith, M.J. 1984. The stability of a dynamic model of traffic assignment- an application of a method of Lyapunov, Transportation Science 18, 245-252.

Smith, M.J. 1984. A descent algorithm for solving monotone variational inequalities and monotone complementarity problems, Journal of optimization theory and applications 44, 485-498.

Williams, J.W.J. 1964. Algorithm 232: Heapsort. Communications of the ACM 7, 701.

Smith, M.J. and Wisten, M.B. 1995. A continuous day-to-day traffic assignment model and the existence of a continuous dynamic user equilibrium, Annals of Operations Research 60, 59-79. 